\title{
Emergência e vigor de mudas de genótipos de mamoeiro em função da irradiância ${ }^{1}$
}

\author{
Liana Hilda Golin Mengarda², José Carlos Lopes² ${ }^{2}$ Rafael Breda Buffon ${ }^{2}$
}

\begin{abstract}
Emergence and vigor of papaya

genotypes seedlings according to irradiance

Luminosity influences seed emergence in the initial development of papaya plants, being necessary the identification of the ideal irradiance level for plantule establishment and formation of vigorous seedlings. This study aimed to evaluate the seed emergence and seedlings vigor of four papaya genotypes, under different irradiance levels. Carica papaya L. seeds, from the Caliman 01 hybrid, Golden THB, triple hybrid and JS12 x Waimanalo hybrid genotypes, were evaluated under four irradiance levels $\left(60 \mu \mathrm{mol} \mathrm{m}^{-2} \mathrm{~s}^{-1}, 130 \mu \mathrm{mol} \mathrm{m}^{-2} \mathrm{~s}^{-1}, 580 \mu \mathrm{mol} \mathrm{m} \mathrm{m}^{-2} \mathrm{~s}^{-1}\right.$ and $\left.1,200 \mu \mathrm{mol} \mathrm{m} \mathrm{m}^{-2} \mathrm{~s}^{-1}\right)$. A completely randomized design, in a $4 \times 4$ split-plot scheme, with four replications, was used. The emergence percentage and speed index were evaluated. The seedlings were measured at 45 and 120 days after sowing (DAS), concerning root and shoot length and dry matter, being calculated the root/shoot ratio and absolute growth rate at $120 \mathrm{DAS}$. The full sun affected emergence, while the low irradiance affected seedling growth. The intermediate irradiance $\left(130 \mu \mathrm{mol} \mathrm{m}^{-2} \mathrm{~s}^{-1}\right.$ and $580 \mu \mathrm{mol} \mathrm{m}^{-2} \mathrm{~s}^{-1}$ ) was more suitable to the development of papaya seedlings up to 120 DAS. The Golden THB genotype was more sensitive to high irradiance, while the Caliman 01 and JS12 x Waimanalo hybrids tolerated the full sun condition.
\end{abstract}

KEY-WORDS: Carica papaya L.; shading; seeds vigor.

\section{INTRODUÇÃO}

O mamoeiro (Carica papaya L.) é uma planta amplamente cultivada em regiões de clima tropical e encontra, no Brasil, condições climáticas favoráveis à sua exploração econômica e alta produtividade. Por ser uma cultura que necessita de renovação dos pomares entre dois e quatro anos, há grande demanda por mudas, justificando pesquisas sobre novas tecnologias para a melhoria das técnicas de produção de sementes, propagação e produção de mudas (Lima et al. 2007, Serrano \& Cattaneo 2010).

\section{RESUMO}

A luminosidade influencia na emergência de sementes e no desenvolvimento inicial do mamoeiro, sendo necessária a identificação do nível de irradiância ideal para o estabelecimento de plântulas e formação de mudas vigorosas. Objetivouse estudar a emergência de sementes e o vigor das mudas de quatro genótipos de mamoeiro, em diferentes níveis de irradiância. Foram utilizadas sementes de Carica papaya L. dos genótipos híbrido Caliman 01, Golden THB, híbrido triplo e híbrido JS12 x Waimanalo, sob quatro níveis de irradiância $\left(60 \mu \mathrm{mol} \mathrm{m} \mathrm{m}^{-2} \mathrm{~s}^{-1}, 130 \mu \mathrm{mol} \mathrm{m} \mathrm{m}^{-2} \mathrm{~s}^{-1}, 580 \mu \mathrm{mol} \mathrm{m} \mathrm{m}^{-2} \mathrm{~s}^{-1} \mathrm{e}\right.$ $\left.1.200 \mu \mathrm{mol} \mathrm{m} \mathrm{m}^{-2} \mathrm{~s}^{-1}\right)$. Utilizou-se delineamento inteiramente casualizado, em esquema de parcelas subdivididas $4 \times 4$, com quatro repetições. Foram avaliados a percentagem e o índice de velocidade de emergência. As mudas foram avaliadas aos 45 e 120 dias após a semeadura (DAS), quanto ao comprimento e massa seca da raiz e da parte aérea, sendo calculadas a razão raiz/parte aérea e a taxa de crescimento absoluto, aos 120 DAS. O sol pleno prejudicou a emergência, enquanto a baixa irradiância prejudicou o desenvolvimento das mudas. A irradiância intermediária $\left(130 \mu \mathrm{mol} \mathrm{m} \mathrm{m}^{-2} \mathrm{~s}^{-1}\right.$ e $\left.580 \mu \mathrm{mol} \mathrm{m}^{-2} \mathrm{~s}^{-1}\right)$ foi mais adequada ao desenvolvimento das mudas de mamoeiro, até os 120 DAS. O genótipo Golden THB foi mais sensível à elevada irradiância, enquanto os híbridos Caliman 01 e JS12 x Waimanalo toleraram a condição de sol pleno.

PALAVRAS-CHAVE: Carica papaya L.; sombreamento; vigor de sementes.

C. papaya é uma planta herbácea de grande porte, que apresenta sensibilidade às variações de temperatura, umidade, défice de pressão de vapor e luminosidade, variações essas que podem afetar os processos fisiológicos (Machado-Filho et al. 2006, Lima et al. 2007, Reis \& Campostrini 2008). A otimização dos fatores do ambiente, durante o cultivo, pode propiciar elevações nas taxas fotossintéticas, podendo contribuir para o aumento da produtividade do mamoeiro (Reis \& Campostrini 2008). Sabe-se, ainda, que o ambiente de cultivo (luminosidade e temperatura) correlaciona-se com a expressão se-

1. Trabalho recebido em set./2013 e aceito para publicação em set./2014 ( $n^{\circ}$ registro: PAT 26429).

2. Universidade Federal do Espírito Santo (UFES), Centro de Ciências Agrárias, Departamento de Produção Vegetal, Alegre, ES, Brasil.E-mails: limengarda@gmail.com, jcufes@bol.com.br, rbredabuffon@gmail.com. 
xual do mamoeiro. Baixa luminosidade associada a elevadas temperaturas favorece a formação de flores estaminadas, indesejáveis (Martelleto et al. 2011).

As variações ambientais também atuam durante as fases inicias do desenvolvimento do mamoeiro, o que sugere o uso de ambientes protegidos na produção de mudas (Araújo et al. 2006, Costa et al. 2010), a fim de proporcionar condições ambientais ideais para a expressão do máximo vigor das sementes.

Trabalhos com sementes de C. papaya vêm sendo realizados, a fim de levantar informações para a produção de sementes de qualidade e para a comparação do desempenho entre materiais genéticos (Cardoso et al. 2009). No entanto, o uso de sementes de boa qualidade não garante a produção de mudas, pois a expressão efetiva do vigor da semente pressupõe ótimas condições ambientais, para o estabelecimento das plântulas. $\mathrm{O}$ crescimento e o rendimento final de um material genético são o resultado de suas interações com o ambiente, sendo necessária, assim, a identificação das condições ambientais ideais para o estabelecimento de plântulas e formação de mudas vigorosas (Marcos Filho 2005, Campostrini \& Glenn 2007).

O estudo das respostas do mamoeiro à ação dos fatores ambientais, como a luminosidade, é importante para minimizar os efeitos indesejados desses sobre os processos fisiológicos, viabilizando novas estratégias de manejo, visando a melhores condições de crescimento e produtividade (Araújo et al. 2006, Lima et al. 2007, Reis \& Campostrini 2008, Costa et al. 2010, Martelleto et al. 2011). Além disso, o entendimento das respostas de genótipos aos fatores específicos do ambiente pode contribuir para programas de melhoramento da espécie (Campostrini \& Glenn 2007). No entanto, são escassos os estudos que relacionam a luminosidade com a emergência e o desenvolvimento inicial. Em vista disso, objetivou-se caracterizar a qualidade física e a emergência das sementes e o vigor das mudas de quatro genótipos de Carica papaya L., em função da irradiância.

\section{MATERIAL E MÉTODOS}

O experimento foi conduzido em casa-de-vegetação, no Centro de Ciências Agrárias da Universidade Federal do Espírito Santo, em Alegre (ES)

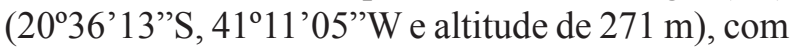
clima caracterizado por inverno seco e verão chuvoso e precipitação média anual de $1.200 \mathrm{~mm}$, entre julho e novembro de 2011.

Foram utilizadas sementes de quatro genótipos de Carica papaya L., fornecidas pela empresa Caliman Agrícola S/A: híbrido Caliman 01, Golden THB, híbrido triplo e híbrido JS12 x Waimanalo. As sementes foram caracterizadas quanto à qualidade física: umidade (U\%), comprimento $(\mathrm{mm})$, diâmetro $(\mathrm{mm})$, peso de mil sementes $(\mathrm{g})$ e número de sementes por $\mathrm{kg}\left(\mathrm{S} \mathrm{kg}^{-1}\right)$, segundo Brasil (2009), com modificações.

A semeadura foi feita em tubetes plásticos, com capacidade para $50 \mathrm{~mL}$, contendo substrato comercial (HS hortaliças, Holambra substratos ${ }^{\circledR}$ ), a 1,5 cm de profundidade. $\mathrm{O}$ experimento foi conduzido em esquema de parcelas subdivididas $4 \times 4$ (quatro níveis de irradiância $\mathrm{x}$ quatro genótipos), com quatro repetições de 25 sementes. $O$ fator irradiância foi obtido com sobreposição de telas sombrite de cor preta (poliolefina) e a irradiância determinada por radiômetro (Light meter LI -250A, LI-COR, USA), sendo observados: $60 \mu \mathrm{mol} \mathrm{m}^{-2} \mathrm{~s}^{-1}$ (três telas sombrite), $130 \mu \mathrm{mol} \mathrm{m}^{-2} \mathrm{~s}^{-1}$ (duas telas sombrite), $580 \mu \mathrm{mol} \mathrm{m} \mathrm{m}^{-2} \mathrm{~s}^{-1}$ (uma tela sombrite) e $1.200 \mu \mathrm{mol} \mathrm{m}{ }^{-2} \mathrm{~s}^{-1}$ (sol pleno).

Foram realizadas duas avaliações do desenvolvimento das plântulas, aos 45 e 120 dias após a semeadura (DAS), utilizando-se quatro repetições de 10 plantas por tratamento. Aos $45 \mathrm{DAS}$, as mudas que foram mantidas sob experimentação para análise aos 120 DAS foram transplantadas para sacos plásticos $(10 \mathrm{~cm} \times 15 \mathrm{~cm})$ contendo o mesmo substrato descrito acima. Durante todo o período de experimentação, foram realizadas irrigações diárias. As variáveis avaliadas foram:

a) Emergência (E) - avaliada diariamente, até a estabilização, considerando-se emersas as plântulas cujos cotilédones encontravam-se acima do substrato;

b) Índice de velocidade de emergência (IVE) conduzido concomitantemente ao teste de emergência, calculado conforme Maguire (1962);

c) Diâmetro do coleto (DC) - determinado na base do caule, com o auxílio de paquímetro digital, após 45 e 120 DAS, sendo o resultado expresso em mm;

d) Comprimento da raiz (CR) e da parte aérea (CPA) - aos 45 e 120 DAS, avaliaram-se o comprimento de raiz primária e parte aérea das plântulas, com o auxílio de uma folha milimetrada, medindo-se da extremidade da raiz até o coleto (CR) e do coleto à extremidade da parte aérea (CPA), sendo os resultados expressos em cm; 
e) Massa seca da raiz (MSR) e da parte aérea (MSPA) - aos 45 e 120 DAS, as plantas foram seccionadas no coleto, raízes e parte aérea, acondicionadas em sacos de papel e mantidas em estufa de circulação forçada de ar, à temperatura de $70^{\circ} \mathrm{C}$, até atingirem massa constante ( 72 horas), sendo pesadas em balança analítica com precisão de $0,0001 \mathrm{~g}$ e os resultados expressos em $\mathrm{mg}$;

f) Taxas de crescimento - a partir das medidas de massa seca realizadas aos $45\left(\mathrm{t}_{1}\right)$ e $120\left(\mathrm{t}_{2}\right)$ DAS, foram calculadas a razão entre massa de raiz e parte aérea (R:PA) e a taxa de crescimento absoluto (TCA = $\left.M_{2}-M_{1} / t_{2}-t_{1}\right)$, conforme descrito por Hunt (1982), em que $M_{1}=$ massa inicial; $M_{2}=$ massa final; $t_{1}=$ tempo inicial; $\mathrm{t}_{2}=$ tempo final.

Os dados em percentagens de emergência foram transformados em arco-seno $\sqrt{x} / 100$ e os dados de índice de velocidade de emergência em $V_{x}+0,5$. Os resultados foram submetidos à análise de variância. A resposta do fator irradiância foi avaliada por análise de regressão, sendo adotados modelos significativos $(\mathrm{p} \leq 0,05)$ e com maior coeficiente de determinação do ajuste da regressão $\left(\mathrm{R}^{2}\right)$. As médias do fator genótipo foram comparadas pelo teste Tukey $(\mathrm{p} \leq 0,05)$.

\section{RESULTADOS E DISCUSSÃO}

Com relação à qualidade física das sementes dos genótipos de mamoeiro, observou-se que os híbridos Caliman 01 e JS12 x Waimanalo apresentaram maior umidade, sendo que Caliman 01 também apresentou maior comprimento e peso de mil sementes (Tabela 1).

A umidade das sementes pode influenciar na velocidade de absorção de água, durante o processo de embebição, sendo que teores de água abaixo dos ideais podem comprometer o processo germinativo. Para mamão, a secagem de sementes até $7,7 \%$ de umidade tem efeito positivo sobre o seu vigor (Berbert et al. 2008), conforme os valores médios de umidade observados para os híbridos Caliman $01 \mathrm{e}$ JS12 x Waimanalo. Em estudos comparativos, a umidade não deve variar mais que $2 \%$ entre as amostras (Marcos Filho 2005). Neste estudo, foi observado variação de $1,62 \%$ entre os genótipos, sendo que o híbrido JS12 x Waimanalo apresentou a maior e o híbrido triplo a menor umidade.

Caliman 01 apresentou maior média de comprimento e peso de mil sementes (Tabela 1). No geral, sementes que apresentam maior tamanho (e massa) também apresentam maior emergência e desenvolvimento inicial das plântulas. Em trabalho realizado com sementes de Tecoma stans, sementes de maior tamanho geraram plântulas mais vigorosas (maior massa seca), principalmente nos três primeiros meses de desenvolvimento (Socolowski et al. 2011). Isso pode estar relacionado à maior disponibilidade de recursos armazenados na semente, para o momento inicial da germinação, quando a plântula não realiza fotossíntese.

Para a percentagem e velocidade de emergência, observou-se interação significativa entre genótipo e irradiância (Tabela 2). No geral, a irradiância baixa e intermediária proporcionaram maior emergência. Para o híbrido Caliman 01 e Golden THB, foi observado ajuste ao modelo quadrático de regressão, com tendência a aumento da emergência, em irradiâncias intermediárias. Para o híbrido triplo, houve ajuste ao modelo linear, sendo observada tendência à redução da emergência, com o aumento da irradiância. As médias do híbrido JS 12 x Waimanalo, em resposta aos níveis de irradiância, foram iguais, não sendo aplicada regressão.

Para $60 \mu \mathrm{mol} \mathrm{m} \mathrm{m}^{-2} \mathrm{~s}^{-1}$ e $580 \mu \mathrm{mol} \mathrm{m} \mathrm{m}^{-2} \mathrm{~s}^{-1}$ de irradiância, o híbrido triplo apresentou menor emergência que os demais genótipos. Para $130 \mu \mathrm{mol} \mathrm{m}^{-2} \mathrm{~s}^{-1}$ de irradiância, os híbridos Caliman 01 e JS12 x

Tabela 1. Características físicas das sementes de diferentes genótipos de C. papaya: umidade (U), comprimento (C), diâmetro (D), peso de mil sementes (PMS) e número de sementes (S) (Alegre, ES, 2011).

\begin{tabular}{|c|c|c|c|c|c|}
\hline \multirow[b]{2}{*}{ Genótipo } & $\mathrm{U}$ & $\mathrm{C}$ & $\mathrm{D}$ & PMS & $\mathrm{S}$ \\
\hline & $\%$ & \multicolumn{2}{|c|}{$\mathrm{mm}$} & $\mathrm{g}$ & $\mathrm{kg}^{-1}$ \\
\hline Caliman 01 & $7,84 \mathrm{a}$ & $5,52 \mathrm{a}$ & $3,05 \mathrm{a}$ & $17,9 \mathrm{a}$ & $5,58 \mathrm{c}$ \\
\hline Golden THB & $6,69 \mathrm{~b}$ & $4,48 \mathrm{c}$ & $3,07 \mathrm{a}$ & $13,4 \mathrm{c}$ & $7,45 \mathrm{a}$ \\
\hline Híbrido triplo & $6,48 \mathrm{~b}$ & $5,00 \mathrm{~b}$ & $2,87 \mathrm{a}$ & $15,3 \mathrm{~b}$ & $6,56 \mathrm{~b}$ \\
\hline JS12 x Waimanalo & $8,10 \mathrm{a}$ & $5,18 \mathrm{~b}$ & $3,16 \mathrm{a}$ & $13,7 \mathrm{c}$ & $7,28 \mathrm{a}$ \\
\hline C.V. $(\%)$ & 5,71 & 4,39 & 5,64 & 5,27 & 5,76 \\
\hline
\end{tabular}

Médias seguidas de letras iguais são agrupadas pelo teste Tukey $(\mathrm{p} \leq 0,05)(\mathrm{n}=4)$. 
Tabela 2. Emergência e índice de velocidade de emergência de plântulas oriundas de sementes de Carica papaya L. submetidas a diferentes níveis de irradiância (Alegre, ES, 2011).

\begin{tabular}{|c|c|c|c|c|c|c|}
\hline \multirow{2}{*}{ Genótipo } & \multicolumn{4}{|c|}{ Irradiância $\left(\mu \mathrm{mol} \mathrm{m}{ }^{-2} \mathrm{~s}^{-1}\right)$} & \multirow{2}{*}{ Equação de regressão } & \multirow{2}{*}{$\mathrm{R}^{2}$} \\
\hline & 60 & 130 & 580 & 1.200 & & \\
\hline \multicolumn{7}{|c|}{ Emergência (\%) } \\
\hline Caliman 01 & $97 \mathrm{a}$ & $100 \mathrm{a}$ & $96 \mathrm{a}$ & $73 \mathrm{~b}$ & $97,4+1,6310^{-2} \mathrm{x}^{\mathrm{ns}}-3,0910^{-5} \mathrm{x}^{2 *}$ & 99,67 \\
\hline Golden THB & $94 \mathrm{a}$ & $78 \mathrm{~b}$ & $84 \mathrm{a}$ & $47 \mathrm{c}$ & $84,2+1,9210^{-2} \mathrm{x}^{\mathrm{ns}}-4,2110^{-5} \mathrm{x}^{2 * *}$ & 94,64 \\
\hline Híbrido triplo & $65 \mathrm{~b}$ & $80 \mathrm{~b}$ & $65 \mathrm{~b}$ & $60 \mathrm{bc}$ & $71,4-9,8910^{-3} \mathrm{x}^{*}$ & 46,75 \\
\hline JS12 x Waimanalo & $91 \mathrm{a}$ & $89 \mathrm{ab}$ & $94 \mathrm{a}$ & $89 \mathrm{a}$ & - & - \\
\hline \multicolumn{7}{|l|}{ C.V. $(\%)=9,91$} \\
\hline \multicolumn{7}{|c|}{ Índice de velocidade de emergência } \\
\hline Caliman 01 & $1,12 \mathrm{a}$ & $1,14 \mathrm{a}$ & $0,54 \mathrm{~b}$ & $0,66 \mathrm{~b}$ & $1,30-2,0010^{-3} \mathrm{x}^{* *}+1,2110^{-6} \mathrm{x}^{2 * *}$ & 96,33 \\
\hline Golden THB & $1,04 \mathrm{a}$ & $0,91 \mathrm{~b}$ & $0,99 \mathrm{a}$ & $0,42 \mathrm{c}$ & $0,95+4,6110^{-4} \mathrm{x}^{\mathrm{ns}}-0,7510^{-7} \mathrm{x}^{2 * *}$ & 94,93 \\
\hline Híbrido triplo & $0,73 \mathrm{~b}$ & $0,92 \mathrm{~b}$ & $0,59 \mathrm{~b}$ & $0,63 \mathrm{~b}$ & $0,82-1,8810^{-4} \mathrm{x}^{* *}$ & 44,29 \\
\hline JS12 x Waimanalo & $1,04 \mathrm{a}$ & $1,04 \mathrm{ab}$ & $1,03 \mathrm{a}$ & $0,93 \mathrm{a}$ & - & - \\
\hline
\end{tabular}

Médias seguidas por mesma letra (genótipos) não diferem entre si, pelo teste Tukey $(\mathrm{p} \leq 0,05)(\mathrm{n}=4) .{ }^{*}, * \mathrm{e}^{\text {ns }}$ : significativo a $1 \%, 5 \%$ e não significativo, respectivamente. $\mathrm{R}^{2}$ = coeficiente de determinação do ajuste da regressão. - médias são estatisticamente iguais e, portanto, não se aplica regressão.

Waimanalo apresentaram maior emergência e, nessa condição, Caliman 01 se destaca por ter apresentado $100 \%$ de germinação. Na condição de sol pleno $\left(1.200 \mu \mathrm{mol} \mathrm{m} \mathrm{m}^{-2} \mathrm{~s}^{-1}\right)$, o híbrido JS12 x Waimanalo apresentou a maior emergência e o genótipo Golden THB a menor emergência (Tabela 2).

Com relação ao índice de velocidade de emergência (IVE), foi observado ajuste ao modelo quadrático de regressão, para o híbrido Caliman 01 e Golden THB. Para o híbrido triplo, houve redução linear da velocidade de germinação, com o aumento da irradiância. Para o híbrido JS12 x Waimanalo, as médias de IVE, em resposta aos níveis de irradiância, foram iguais (Tabela 2). Observou-se que o híbrido triplo apresentou menor média, com relação aos demais genótipos, em $60 \mu \mathrm{mol} \mathrm{m}{ }^{-2} \mathrm{~s}^{-1}$ de irradiância. $\mathrm{O}$ híbrido JS12 x Waimanalo apresentou maior média na condição de sol pleno $\left(1.200 \mu \mathrm{mol} \mathrm{m}^{-2} \mathrm{~s}^{-1}\right)$ (Tabela 2).

Em geral, observou-se que o sol pleno (1.200 $\mu \mathrm{mol} \mathrm{m} \mathrm{m}^{-2} \mathrm{~s}^{-1}$ de irradiância) foi prejudicial ao processo germinativo. Segundo Carvalho \& Nakagawa (2012), temperaturas inferiores ou superiores à ótima (associadas à menor e maior luminosidade) tendem a reduzir a velocidade de germinação, expondo as plântulas a fatores adversos por maior período.

Os híbridos Caliman 01 e JS12 x Waimanalo apresentaram médias de emergência e IVE superiores aos demais genótipos, nos diferentes níveis de irradiância. A resposta diferenciada entre os genótipos pode ser atribuída às suas características genéticas. Mesmo quando fornecidas condições idênticas para a germinação, algumas sementes originam plântulas com maior taxa de crescimento, em função de apresentarem maior capacidade de transformação do suprimento de reservas dos tecidos de armazenamento e maior incorporação desses pelo eixo embrionário (Marcos Filho 2005).

Ainda em função de o híbrido Caliman 01 apresentar maior tamanho de sementes (comprimento e PMS), associado à boa qualidade fisiológica (emergência e IVE), as respostas germinativas dos genótipos de mamoeiro parecem ter relação com a qualidade física das sementes. Socolowski et al. (2011) afirmam que, enquanto a emergência das plântulas está diretamente relacionada à massa das sementes, a sobrevivência das plântulas está relacionada à presença da luz. Assim, as diferenças podem ser visíveis nos momentos iniciais do desenvolvimento e, após a obtenção do estande de plântulas, existe tendência de uniformidade entre as mudas.

Com relação ao desenvolvimento inicial das plântulas, avaliado aos 45 e 120 DAS, houve alterações significativas, quanto ao crescimento e incremento de biomassa das plântulas dos genótipos de mamão, em diferentes irradiâncias, sendo observada interação significativa entre os fatores genótipo e irradiância (Tabelas 3 e 4).

De acordo com a avaliação aos 45 DAS, a irradiância de $60 \mu \mathrm{mol} \mathrm{m} \mathrm{m}^{-2} \mathrm{~s}^{-1}$ prejudicou o desenvolvimento das raízes, enquanto o sol pleno $\left(1.200 \mu \mathrm{mol} \mathrm{m} \mathrm{m}^{-2} \mathrm{~s}^{-1}\right)$ reduziu o desenvolvimento da parte aérea. Para $1.200 \mu \mathrm{mol} \mathrm{m}{ }^{-2} \mathrm{~s}^{-1}$ de irradiância, o genótipo Golden THB foi o mais prejudicado, quanto 
Tabela 3. Médias das variáveis de crescimento inicial das plântulas de Carica papaya L. submetidas a diferentes níveis de irradiância, aos 45 dias após a semeadura (Alegre, ES, 2011).

\begin{tabular}{|c|c|c|c|c|c|c|}
\hline \multirow{2}{*}{ Genótipo } & \multicolumn{4}{|c|}{ Irradiância $\left(\mu \mathrm{mol} \mathrm{m} \mathrm{m}^{-2} \mathrm{~s}^{-1}\right)$} & \multirow{2}{*}{ Equação de regressão } & \multirow{2}{*}{$\mathrm{R}^{2}$} \\
\hline & 60 & 130 & 580 & 1.200 & & \\
\hline \multicolumn{7}{|c|}{ Comprimento da raiz (cm) } \\
\hline Caliman 01 & $11,4 \mathrm{a}$ & $13,1 \mathrm{ab}$ & $14,2 \mathrm{a}$ & $13,9 \mathrm{a}$ & $11,5+810^{-3} \mathrm{x}^{* *}-510^{-6} \mathrm{x}^{2 * *}$ & 82,88 \\
\hline Golden THB & $11,9 \mathrm{a}$ & $13,7 \mathrm{a}$ & $14,0 \mathrm{ab}$ & $12,7 \mathrm{~b}$ & $12,2+610^{-3} \mathrm{x}^{* *}-510^{-6} \mathrm{x}^{2 * *}$ & 59,43 \\
\hline Híbrido triplo & $11,4 \mathrm{a}$ & $12,6 \mathrm{~b}$ & $13,2 \mathrm{~b}$ & $13,9 \mathrm{a}$ & $11,9+1,8210^{-3} \mathrm{x}^{* *}$ & 80,46 \\
\hline JS12 x Waimanalo & $11,4 \mathrm{a}$ & $12,2 \mathrm{~b}$ & $13,2 \mathrm{~b}$ & $13,9 \mathrm{a}$ & $11,7+210^{-3} \mathrm{x}^{* *}$ & 89,99 \\
\hline \multicolumn{7}{|l|}{ C.V. $(\%)=4,32$} \\
\hline \multicolumn{7}{|c|}{ Comprimento da parte aérea $(\mathrm{cm})$} \\
\hline Caliman 01 & $13,1 \mathrm{a}$ & $16,0 \mathrm{a}$ & $10,5 \mathrm{a}$ & $6,2 \mathrm{a}$ & $15,16-7,4510^{-3} \mathrm{x}^{* *}$ & 88,48 \\
\hline Golden THB & $12,3 \mathrm{ab}$ & $11,6 \mathrm{c}$ & $10,3 \mathrm{ab}$ & $5,5 \mathrm{a}$ & $12,2-1,410^{-3} \mathrm{x}^{\mathrm{ns}}-310^{-6} \mathrm{x}^{2 * *}$ & 99,29 \\
\hline Híbrido triplo & $11,6 \mathrm{bc}$ & $13,2 \mathrm{~b}$ & $9,3 \mathrm{bc}$ & $5,9 \mathrm{a}$ & $12,88-5,8410^{-3} \mathrm{x}^{* *}$ & 93,62 \\
\hline JS12 x Waimanalo & $10,7 \mathrm{c}$ & $11,7 \mathrm{c}$ & $9,0 \mathrm{c}$ & $6,7 \mathrm{a}$ & $11,50-4,0210^{-3} \mathrm{x}^{* *}$ & 94,43 \\
\hline \multicolumn{7}{|l|}{ C.V. $(\%)=6,04$} \\
\hline \multicolumn{7}{|c|}{ Diâmetro do coleto $(\mathrm{mm})$} \\
\hline Caliman 01 & $0,29 \mathrm{a}$ & $0,33 \mathrm{a}$ & $0,33 \mathrm{a}$ & $0,21 \mathrm{~b}$ & $0,3+1,910^{-4} x^{* *}-210^{-7} x^{2 * *}$ & 95,66 \\
\hline Golden THB & $0,29 \mathrm{a}$ & $0,25 \mathrm{~b}$ & $0,30 \mathrm{a}$ & $0,16 \mathrm{c}$ & $0,3+1,810^{-4} \mathrm{x}^{* *}-210^{-7} \mathrm{x}^{2 * *}$ & 84,47 \\
\hline Híbrido triplo & $0,28 \mathrm{a}$ & $0,29 \mathrm{ab}$ & $0,30 \mathrm{a}$ & $0,20 \mathrm{~b}$ & $0,3+1,510^{-4} x^{*}-210^{-7} x^{2 * *}$ & 99,28 \\
\hline JS12 x Waimanalo & $0,25 \mathrm{a}$ & $0,27 \mathrm{~b}$ & $0,29 \mathrm{a}$ & $0,26 \mathrm{a}$ & - & - \\
\hline \multicolumn{7}{|l|}{ C.V. $(\%)=8,70$} \\
\hline \multicolumn{7}{|c|}{ Massa seca da raiz (mg) } \\
\hline Caliman 01 & $8,1 \mathrm{a}$ & $20,1 \mathrm{a}$ & $29,9 \mathrm{a}$ & $16,8 \mathrm{~b}$ & $7,6+7,110^{-2} \mathrm{X}^{* *}-510^{-5} \mathrm{x}^{2 * *}$ & 87,30 \\
\hline Golden THB & $6,3 \mathrm{a}$ & $15,5 \mathrm{~b}$ & $22,0 \mathrm{~b}$ & $8,3 \mathrm{c}$ & $5,9+5,510^{-2} \mathrm{X}^{* *}-410^{-5} \mathrm{x}^{2 * *}$ & 88,14 \\
\hline Híbrido triplo & $6,4 \mathrm{a}$ & $14,4 \mathrm{~b}$ & $23,0 \mathrm{~b}$ & $15,8 \mathrm{~b}$ & $5,6+5,210^{-2} \mathrm{x}^{* *}-410^{-5} \mathrm{x}^{2 * *}$ & 91,16 \\
\hline JS12 x Waimanalo & $5,3 \mathrm{a}$ & $14,7 \mathrm{~b}$ & $24,7 \mathrm{~b}$ & $26,4 \mathrm{a}$ & $5,1+5,210^{-2} \mathrm{x}^{* *}-310^{-5} \mathrm{x}^{2 * *}$ & 93,29 \\
\hline \multicolumn{7}{|l|}{ C.V. $(\%)=12,59$} \\
\hline \multicolumn{7}{|c|}{ Massa seca da parte aérea (mg) } \\
\hline Caliman 01 & $51,4 \mathrm{a}$ & $98,3 \mathrm{a}$ & $87,9 \mathrm{a}$ & $36,8 \mathrm{~b}$ & $62,4+0,12 x^{* *}-1,210^{-4} x^{2 * *}$ & 67,47 \\
\hline Golden THB & $41,4 \mathrm{a}$ & $57,7 \mathrm{c}$ & $64,9 \mathrm{~b}$ & $20,2 \mathrm{c}$ & $40,3+0,10 \mathrm{x} * *-1010^{-4} \mathrm{x}^{2 * *}$ & 95,11 \\
\hline Híbrido triplo & $45,8 \mathrm{a}$ & $75,6 \mathrm{~b}$ & $69,0 \mathrm{~b}$ & $32,1 \mathrm{bc}$ & $52,4+0,08 x^{* *}-8,310^{-5} x^{2 * *}$ & 73,15 \\
\hline JS12 x Waimanalo & $36,7 \mathrm{a}$ & $64,5 \mathrm{bc}$ & $71,5 \mathrm{~b}$ & $64,8 \mathrm{a}$ & $41,3+0,09 x^{* *}-6,110^{-5} x^{2 * *}$ & 64,37 \\
\hline C.V. $(\%)=13,73$ & & & & & & \\
\hline
\end{tabular}

ao comprimento das raízes, diâmetro do coleto e massa seca das raízes, enquanto o genótipo Híbrido JS12 x Waimanalo apresentou maior massa seca da raiz e da parte aérea e diâmetro do coleto. Caliman 01 apresentou maior comprimento e massa seca da parte aérea, em irradiância intermediária $\left(130 \mu \mathrm{mol} \mathrm{m}^{-2} \mathrm{~s}^{-1}\right.$ e $580 \mu \mathrm{mol} \mathrm{m}^{-2} \mathrm{~s}^{-1}$ ) (Tabela 3).

Aos 120DAS, abaixa irradiância $\left(60 \mu \mathrm{molm}^{-2} \mathrm{~s}^{-1}\right)$ influenciou negativamente no desenvolvimento das mudas. Para todas as características avaliadas, em todos os genótipos, foi observada resposta ajustada ao modelo quadrático, com tendência ao aumento do desenvolvimento das plântulas sob irradiâncias intermediárias $\left(130 \mu \mathrm{mol} \mathrm{m} \mathrm{m}^{-2} \mathrm{~s}^{-1}\right.$ e $\left.580 \mu \mathrm{mol} \mathrm{m}^{-2} \mathrm{~s}^{-1}\right)$ e redução na irradiância baixa e alta $\left(60 \mu \mathrm{mol} \mathrm{m}{ }^{-2} \mathrm{~s}^{-1}\right.$ e $1.200 \mu \mathrm{mol} \mathrm{m}{ }^{-2} \mathrm{~s}^{-1}$ ) (Tabela 4).
Para a maioria dos genótipos, houve menor desenvolvimento da parte aérea (CPA) no tratamento a sol pleno, o que pode estar relacionado aos mecanismos de dissipação do excesso de energia luminosa (Campostrini \& Glenn 2007, Reis \& Campostini 2008), sugerindo a necessidade de sombreamento, durante o desenvolvimento inicial. Aos 45 DAS, no entanto, a pleno sol $\left(1.200 \mu \mathrm{mol} \mathrm{m} \mathrm{m}^{-2} \mathrm{~s}^{-1}\right)$, o híbrido JS12 x Waimanalo apresentou maiores médias de diâmetro do coleto e massa seca das plântulas, o que pode indicar que esse genótipo apresenta um mecanismo eficiente de dispersão do excesso de luz pelos fotossistemas, evitando fotodanos e maximizando a eficiência fotossintética e o crescimento, nessa condição (Hunt 1982, Campostrini \& Glenn 2007, Lima et al. 2007). 
Tabela 4. Médias das variáveis de crescimento inicial das mudas de Carica papaya L. submetidas a diferentes níveis de irradiância, aos 120 dias após a semeadura (Alegre, ES, 2011).

\begin{tabular}{|c|c|c|c|c|c|c|}
\hline \multirow{2}{*}{ Genótipo } & \multicolumn{4}{|c|}{ Irradiância $\left(\mu \mathrm{mol} \mathrm{m}{ }^{-2} \mathrm{~s}^{-1}\right)$} & \multirow{2}{*}{ Equação de regressão } & \multirow{2}{*}{$\mathrm{R}^{2}$} \\
\hline & 60 & 130 & 580 & 1.200 & & \\
\hline \multicolumn{7}{|c|}{ Comprimento da raiz $(\mathrm{cm})$} \\
\hline Caliman 01 & $16,1 \mathrm{a}$ & $17,9 \mathrm{~b}$ & $28,8 \mathrm{a}$ & $27,8 \mathrm{a}$ & $13,5+3,910^{-2} x^{* *}-2,310^{-5} \mathrm{x}^{2 * *}$ & 99,81 \\
\hline Golden THB & $15,8 \mathrm{a}$ & $16,6 \mathrm{~b}$ & $29,8 \mathrm{a}$ & $22,8 \mathrm{~b}$ & $11,9+5,110^{-2} \mathrm{x}^{* *}-3,510^{-5} \mathrm{x}^{2 * *}$ & 98,03 \\
\hline Híbrido triplo & $17,5 \mathrm{a}$ & $23,5 \mathrm{a}$ & $28,9 \mathrm{a}$ & $23,4 \mathrm{~b}$ & $17,1+3,610^{-2} \mathrm{x}^{* *}-2,610^{-5} \mathrm{x}^{2 * *}$ & 88,13 \\
\hline JS12 x Waimanalo & $16,7 \mathrm{a}$ & $18,6 \mathrm{~b}$ & $26,4 \mathrm{a}$ & $29,6 \mathrm{a}$ & $15,3+2,610^{-2} \mathrm{x}^{* *}-1,210^{-5} \mathrm{x}^{2 * *}$ & 99,97 \\
\hline \multicolumn{7}{|l|}{ C.V. $(\%)=9,93$} \\
\hline \multicolumn{7}{|c|}{ Comprimento da parte aérea $(\mathrm{cm})$} \\
\hline Caliman 01 & $38,5 \mathrm{a}$ & $60,3 \mathrm{a}$ & $55,3 \mathrm{a}$ & 29,9 a & $43,5+5,810^{-2} \mathrm{x}^{* *}-5,810^{-5} \mathrm{x}^{2 * *}$ & 70,80 \\
\hline Golden THB & $39,5 \mathrm{a}$ & $46,2 \mathrm{~b}$ & $60,2 \mathrm{a}$ & $16,0 \mathrm{~b}$ & $34,2+0,10 x * *-9,710^{-5} x^{2 * *}$ & 99,96 \\
\hline Híbrido triplo & $44,7 \mathrm{a}$ & $60,2 \mathrm{a}$ & 52,6 a & $19,7 \mathrm{~b}$ & $48,3+4,310^{-2} \mathrm{x}^{* *}-5,610^{-5} \mathrm{x}^{2 * *}$ & 90,03 \\
\hline JS12 x Waimanalo & $38,2 \mathrm{a}$ & $50,7 \mathrm{~b}$ & $42,3 \mathrm{~b}$ & $22,5 \mathrm{ab}$ & $42,2+2,110^{-2} \mathrm{x}^{\mathrm{ns}}-3,110^{-5} \mathrm{x}^{2 * *}$ & 83,63 \\
\hline \multicolumn{7}{|l|}{ C.V. $(\%)=10,95$} \\
\hline \multicolumn{7}{|c|}{ Diâmetro do coleto $(\mathrm{mm})$} \\
\hline Caliman 01 & $0,60 \mathrm{a}$ & $0,77 \mathrm{a}$ & $0,86 \mathrm{a}$ & $0,75 \mathrm{a}$ & $0,6+7,510^{-3} \mathrm{x}^{* *}-5,610^{-8} \mathrm{x}^{2 * *}$ & 78,53 \\
\hline Golden THB & $0,58 \mathrm{a}$ & $0,60 \mathrm{~b}$ & $0,80 \mathrm{a}$ & $0,53 \mathrm{~b}$ & $0,5+9,410^{-4} \mathrm{x}^{* *}-7,710^{-8} \mathrm{x}^{2 * *}$ & 98,00 \\
\hline Híbrido triplo & $0,66 \mathrm{a}$ & $0,78 \mathrm{a}$ & $0,80 \mathrm{a}$ & $0,68 \mathrm{a}$ & $0,7+4,510^{-4} x^{* *}-3,710^{-7} x^{2 * *}$ & 68,65 \\
\hline JS12 x Waimanalo & $0,62 \mathrm{a}$ & $0,75 \mathrm{a}$ & $0,74 \mathrm{a}$ & $0,67 \mathrm{a}$ & $0,7+3,410^{-4} x^{*}-2,710^{-7} x^{2 *}$ & 46,58 \\
\hline \multicolumn{7}{|l|}{ C.V. $(\%)=8,98$} \\
\hline \multicolumn{7}{|c|}{ Massa seca da raiz (mg) } \\
\hline Caliman 01 & $42,5 \mathrm{a}$ & $211 \mathrm{a}$ & $528 \mathrm{a}$ & $464 \mathrm{a}$ & $-6,15+1,45 x^{* *}-8,8510^{-5} x^{2 * *}$ & 97,88 \\
\hline Golden THB & 43,5 a & $59,2 \mathrm{~b}$ & $459 \mathrm{ab}$ & $167 \mathrm{c}$ & $-82,7+1,58 x^{* *}-1,1410^{-3} x^{2 * *}$ & 97,03 \\
\hline Híbrido triplo & 92,5 a & $203 \mathrm{a}$ & 509 a & $371 \mathrm{~b}$ & $27,1+1,35 x^{* *}-8,8710^{-4} x^{2 * *}$ & 99,60 \\
\hline JS12 x Waimanalo & $74,5 \mathrm{a}$ & $221 \mathrm{a}$ & $395 \mathrm{~b}$ & $422 \mathrm{ab}$ & $65,7+0,86 x^{* *}-4,7610^{-4} x^{2 * *}$ & 94,42 \\
\hline \multicolumn{7}{|l|}{ C.V. $(\%)=17,53$} \\
\hline \multicolumn{7}{|c|}{ Massa seca da parte aérea (mg) } \\
\hline Caliman 01 & $488 \mathrm{a}$ & $1.287 \mathrm{a}$ & $1.762 \mathrm{a}$ & $1.266 \mathrm{a}$ & $524+3,8 x^{* *}-2,6710^{-3} x^{2 * *}$ & 79,79 \\
\hline Golden THB & $430 \mathrm{a}$ & $665 \mathrm{~b}$ & $1.721 \mathrm{a}$ & $447 \mathrm{c}$ & $109+5,1 x^{* *}-4,0010^{-3} \mathrm{x}^{2 * *}$ & 99,79 \\
\hline Híbrido triplo & $667 \mathrm{a}$ & $1.244 \mathrm{a}$ & $1.714 \mathrm{a}$ & $747 \mathrm{~b}$ & $616+3,7 \mathrm{x} * *-2,9810^{-3} \mathrm{x}^{2 * *}$ & 90,32 \\
\hline JS12 $\mathrm{x}$ Waimanalo & $442 \mathrm{a}$ & $1.317 \mathrm{a}$ & $1.152 \mathrm{~b}$ & $679 \mathrm{bc}$ & $673+1,9 x^{* *}-1,6210^{-3} x^{2 * *}$ & 38,99 \\
\hline C.V. $(\%)=14,42$ & & & & & & \\
\hline
\end{tabular}

Reis \& Campostrini (2008) investigaram a eficiência fotoquímica do mamoeiro em campo, em duas épocas do ano, verificando que a menor assimilação fotossintética teve relação com a maior luminosidade (dias com ausência de nuvens). Embora a luz insuficiente, ou em excesso, possa causar comprometimentos no processo fotossintético, as plantas possuem mecanismos para evitar os danos fotoinibitórios.

No entanto, dependendo da intensidade da irradiância imposta, os processos fotossintéticos podem ser comprometidos e, consequentemente, ocorrer menor incremento de biomassa das plantas. Como resposta, as plantas investem mais no desenvolvimento da parte aérea e na superfície foliar, mas de forma desequilibrada, levando à formação de plantas estioladas e frágeis (Hunt 1982) e mudas sem padrão adequado para serem levadas a campo. Dessa forma, o aumento do comprimento da parte aérea (CPA), sob baixa irradiância $\left(60 \mu \mathrm{mol} \mathrm{m} \mathrm{m}^{-2} \mathrm{~s}^{-1}\right)$, pode indicar uma fuga à condição estressante, pela falta de luz, e não maior desenvolvimento inicial das mudas. Assim, devem ser observadas outras características, como o diâmetro do coleto, razão entre a massa da raiz e da parte aérea e taxas de crescimento.

Em relação ao diâmetro do coleto (DC), foram observadas respostas ajustadas ao modelo quadrático, com aumento nas irradiâncias intermediárias $\left(130 \mu \mathrm{mol} \mathrm{m} \mathrm{m}^{-2} \mathrm{~s}^{-1}\right.$ e $\left.580 \mu \mathrm{mol} \mathrm{m} \mathrm{m}^{-2} \mathrm{~s}^{-1}\right)$ para todos os genótipos, à exceção do híbrido JS12 x Waimanalo, na avaliação aos $45 \mathrm{DAS}$, que manteve média constante. 
Neste estudo, observou-se que, até os 120 DAS (quatro meses de cultivo), os genótipos de mamão avaliados apresentaram maior germinação e desenvolvimento inicial das plantas, em irradiância intermediária. No entanto, após 12 meses de cultivo, Martelleto et al. (2008) verificaram maior diâmetro do tronco do mamoeiro, em tratamentos a sol pleno, com diâmetro $15 \%$ superior, em relação ao sombreamento. Isso sugere a necessidade de sombreamento nas fases iniciais do estabelecimento das plântulas (germinação), mas indica que o sombreamento intenso pode ser prejudicial, no decorrer do seu cultivo (desenvolvimento das mudas). Como plantas com maior diâmetro de coleto são muito importantes, do ponto de vista agronômico, tendo-se em vista a estreita correlação entre diâmetro do tronco e produção de frutos, no mamoeiro (Ferreira et al. 2012), é necessário o aumento gradual dos níveis de irradiância, para que tal relação se estabeleça e mudas mais vigorosas possam ser produzidas.

O aumento dos níveis de irradiância também é imprescindível, porque as condições ambientais influenciam em processos fisiológicos capazes de alterar a expressão sexual do mamoeiro: menor irradiância induz à formação de flores estaminadas (plantas masculinas, indesejáveis) (Martelleto et al. 2011).

Aos 45 e 120 DAS, para todos os genótipos estudados, observou-se tendência a maiores médias de massa seca das plântulas, em irradiâncias intermediárias (Tabelas 3 e 4), assim como maiores relações entre massa seca das raízes e da parte aérea
(Tabela 5). Em geral, a relação entre a massa da parte radicular $(\mathrm{R})$ deve ser próxima à da parte aérea $(\mathrm{PA})$, para que a muda tenha um bom desenvolvimento no campo (Hunt 1982). Além disso, a variação da relação R/PA é um indicativo da capacidade de os genótipos se ajustarem à condição de sombreamento.

Para os genótipos de mamoeiro avaliados neste estudo, a relação R/PA sugere maior alocação de massa seca para a parte aérea das plantas sob irradiância baixa $\left(60 \mu \mathrm{mol} \mathrm{m} \mathrm{m}^{-2} \mathrm{~s}^{-1}\right)$, com menores médias de R/PA, quando comparada aos demais níveis de irradiância (Tabela 5). Essa resposta pode representar prejuízo ao desenvolvimento das mudas e, com isso, novamente observa-se tendência à necessidade de redução gradual do sombreamento, durante a produção de mudas de mamoeiro (Lima et al. 2007, Martelleto et al. 2008).

Lima et al. (2007) avaliaram índices fisiológicos, como taxas de crescimento relativo e absoluto, de mudas de mamoeiro, em casa-de-vegetação. Tais índices mostraram-se ferramentas eficientes para identificar e comparar diferentes materiais genéticos, durante o crescimento inicial de plantas de mamoeiro. Essas análises são um instrumento simples e preciso, para avaliar as respostas fisiológicas, pois, assim, é possível conhecer a cinética de produção de biomassa das plantas (Benincasa 2003).

No presente estudo, a avaliação da taxa de crescimento absoluto dos genótipos indica que as irradiâncias intermediárias $\left(130 \mu \mathrm{mol} \mathrm{m} \mathrm{m}^{-2} \mathrm{~s}^{-1} \mathrm{e}\right.$ $580 \mu \mathrm{mol} \mathrm{m}^{-2} \mathrm{~s}^{-1}$ ) promoveram maior incremento de biomassa nas mudas. Taxa de crescimento absoluto

Tabela 5. Razão entre massa seca da raiz e da parte aérea e taxa de crescimento absoluto de plântulas oriundas de sementes de Carica papaya L. submetidas a diferentes níveis de irradiância (Alegre, ES, 2011).

\begin{tabular}{|c|c|c|c|c|c|c|}
\hline \multirow{2}{*}{ Genótipo } & \multicolumn{4}{|c|}{ Irradiância $\left(\mu \mathrm{mol} \mathrm{m}{ }^{-2} \mathrm{~s}^{-1}\right)$} & \multirow{2}{*}{ Equação de regressão } & \multirow{2}{*}{$\mathrm{R}^{2}$} \\
\hline & 60 & 130 & 580 & 1.200 & & \\
\hline \multicolumn{7}{|c|}{ Razão raiz/parte aérea } \\
\hline Caliman 01 & $0,10 \mathrm{~b}$ & $0,16 \mathrm{a}$ & $0,28 \mathrm{bc}$ & $0,40 \mathrm{~b}$ & $0,1+410^{-4} x^{* *}-1,110^{-7} x^{2 * *}$ & 98,72 \\
\hline Golden THB & $0,10 \mathrm{~b}$ & $0,10 \mathrm{~b}$ & $0,27 \mathrm{c}$ & $0,52 \mathrm{a}$ & $0,08+310^{-4} \mathrm{x}^{* *}-0,710^{-7} \mathrm{x}^{2 * *}$ & 99,83 \\
\hline Híbrido triplo & $0,14 \mathrm{ab}$ & $0,17 \mathrm{a}$ & $0,32 \mathrm{ab}$ & $0,52 \mathrm{a}$ & $0,12+3,3410^{-4} \mathrm{x}^{* *}$ & 99,98 \\
\hline JS12 x Waimanalo & $0,16 \mathrm{a}$ & $0,18 \mathrm{a}$ & $0,34 \mathrm{a}$ & $0,50 \mathrm{a}$ & $0,14+410^{-4} \mathrm{x} * *-0,810^{-7} \mathrm{x}^{2 * *}$ & 99,98 \\
\hline \multicolumn{7}{|l|}{ C.V. $(\%)=8,32$} \\
\hline \multicolumn{7}{|c|}{ Taxa de crescimento absoluto ( $m g$ dia $\left.{ }^{-1}\right)$} \\
\hline Caliman 01 & $6,28 \mathrm{a}$ & $18,40 \mathrm{a}$ & $28,96 \mathrm{a}$ & $22,34 \mathrm{a}$ & $6+6,810^{-2} x^{* *}-4,510^{-5} x^{2 * *}$ & 87,77 \\
\hline Golden THB & $5,68 \mathrm{a}$ & $8,68 \mathrm{~b}$ & $27,90 \mathrm{a}$ & $7,80 \mathrm{c}$ & $-0,3+0,1 x^{* *}-6,710^{-5} x^{2 * *}$ & 99,23 \\
\hline Híbrido triplo & $9,42 \mathrm{a}$ & $18,09 \mathrm{a}$ & $28,40 \mathrm{a}$ & $14,62 \mathrm{~b}$ & $7,8+0,07 x^{* *}-510^{-5} x^{2 * *}$ & 93,90 \\
\hline JS12 x Waimanalo & $6,34 \mathrm{a}$ & $19,44 \mathrm{a}$ & $19,32 \mathrm{~b}$ & $13,46 \mathrm{~b}$ & $9,2+0,04 x^{* *}-2,710^{-5} x^{2 * *}$ & 45,30 \\
\hline
\end{tabular}

C.V. $(\%)=14,45$

Médias seguidas por mesma letra (genótipos) não diferem ente si, pelo teste Tukey $(\mathrm{p} \leq 0,05)(\mathrm{n}=4)$. **, * e ns: significativo a $1 \%, 5 \%$ e não significativo, respectivamente. $\mathrm{R}^{2}=$ coeficiente de determinação do ajuste da regressão. 
inferior foi verificada para todos os genótipos, em $60 \mu \mathrm{mol} \mathrm{m} \mathrm{s}^{-2} \mathrm{~s}^{-1}$ (Tabela 5).

O genótipo Caliman 01 apresentou maior crescimento, em relação aos demais genótipos, em $1.200 \mu \mathrm{mol} \mathrm{m}{ }^{-2} \mathrm{~s}^{-1}$ de irradiância, enquanto o genótipo Golden THB apresentou taxas de crescimento inferiores aos demais genótipos, em $130 \mu \mathrm{mol} \mathrm{m}^{-2} \mathrm{~s}^{-1}$ e $1.200 \mu \mathrm{mol} \mathrm{m}^{-2} \mathrm{~s}^{-1}$ (Tabela 5).

Variação da massa da raiz, em relação à parte aérea, e da taxa de crescimento absoluto podem ser empregadas para descrever as preferências ecológicas das plantas, quanto à disponibilidade de luz (Lambers \& Poorter 1992). A análise de crescimento tem sido usada na tentativa de explicar diferenças no crescimento de ordem genética, ou resultante de modificações do ambiente, e constitui ferramenta eficiente para a identificação de materiais promissores, além de identificar características que, no crescimento inicial, indiquem possibilidade de aumento no rendimento da planta adulta, favorecendo trabalhos de melhoramento, na busca por materiais mais produtivos (Lima et al. 2007).

Com base nessas análises, é notória a influência da luminosidade no desenvolvimento inicial dos genótipos de mamoeiro: as irradiâncias intermediárias $\left(130 \mu \mathrm{mol} \mathrm{m}^{-2} \mathrm{~s}^{-1}\right.$ e $\left.580 \mu \mathrm{mol} \mathrm{m} \mathrm{m}^{-2} \mathrm{~s}^{-1}\right)$ promoveram bom desempenho germinativo e desenvolvimento das mudas. Os extremos de irradiância $\left(60 \mu \mathrm{mol} \mathrm{m}^{-2} \mathrm{~s}^{-1} \mathrm{e}\right.$ $1.200 \mu \mathrm{mol} \mathrm{m} \mathrm{m}^{-2} \mathrm{~s}^{-1}$ ) se mostraram prejudiciais, uma vez que, nos momentos iniciais do desenvolvimento, o sol pleno prejudica a emergência e, durante o desenvolvimento das mudas, a baixa irradiância reduz acentuadamente o desenvolvimento radicular, em relação ao da parte aérea, o diâmetro do coleto das mudas e a taxa de crescimento absoluto.

\section{CONCLUSÕES}

1. A elevada irradiância $\left(1.200 \mu \mathrm{mol} \mathrm{m} \mathrm{m}^{-2} \mathrm{~s}^{-1}\right)$ prejudicou a emergência e a baixa irradiância $\left(60 \mu \mathrm{mol} \mathrm{m} \mathrm{m}^{-2} \mathrm{~s}^{-1}\right)$ o desenvolvimento das mudas.

2. As irradiâncias intermediárias $\left(130 \mu \mathrm{mol} \mathrm{m}^{-2} \mathrm{~s}^{-1}\right.$ e $\left.580 \mu \mathrm{mol} \mathrm{m} \mathrm{m}^{-2} \mathrm{~s}^{-1}\right)$ mostraram-se adequadas à emergência e desenvolvimento das mudas, até os 120 dias.

3. O genótipo Golden THB foi o mais sensível à condição de sol pleno, enquanto os genótipos Caliman 01 e o híbrido JS12 x Waimanalo toleraram a luminosidade mais intensa, durante a emergência e o desenvolvimento inicial das mudas.

\section{AGRADECIMENTOS}

À Caliman Agrícola S/A, pela cessão de sementes, à Coordenação de Aperfeiçoamento de Pessoal de Nível Superior (Capes) e ao Conselho Nacional de Desenvolvimento Científico e Tecnológico (CNPq), pela concessão de bolsas de estudo e pesquisa, respectivamente.

\section{REFERÊNCIAS}

ARAÚJO, J. G. et al. Efeito do recipiente e ambiente de cultivo sobre o desenvolvimento de mudas de mamoeiro cv. Sunrise Solo. Revista Brasileira de Fruticultura, Jaboticabal, v. 28, n. 3, p. 526-529, 2006.

BERBERT, P. A. et al. Qualidade fisiológica de semente de mamão em função da secagem e do armazenamento. Revista Brasileira de Sementes, Londrina, v. 30, n. 1, p. 40-48, 2008.

BENINCASA, M. M. P. Análise de crescimento de plantas. 2. ed. Jaboticabal: Funep, 2003.

BRASIL. Ministério da Agricultura, Pecuária e Abastecimento. Regras para análise de sementes. Brasília, DF: MAPA/DAS/ACS, 2009.

CAMPOSTRINI, E.; GLENN, D. M. Ecophysiology of papaya: a review. Brazilian Journal of Plant Physiology, Campos dos Goytacazes, v. 19, n. 4, p. 413-424, 2007.

CARDOSO, D. L. et al. Diversidade genética e parâmetros genéticos relacionados à qualidade fisiológica de sementes em germoplasma de mamoeiro. Revista Ceres, Viçosa, v. 56, n. 5, p. 572-579, 2009.

CARVALHO, N. M.; NAKAGAWA, J. Sementes: ciência, tecnologia e produção. 5. ed. Campinas: Funep, 2012.

COSTA, E. et al. Crescimento de mudas de mamoeiro conduzidas em diferentes ambientes protegidos, recipientes e substratos na região de Aquidauana, Estado do Mato Grosso do Sul. Acta Scientiarum Agronomy, Maringá, v. 32, n. 3, p. 463-470, 2010.

FERREIRA, J. P. et al. Correlações entre características morfoagronômicas de acessos de mamoeiro. Enciclopédia Biosfera, Goiânia, v. 8, n. 14, p. 246-257, 2012.

HUNT, R. Plant growth curves: the functional approach to plant growth analysis. London: Edward Arnold Publishers, 1982.

LAMBERS, H.; POORTER, H. Inherent variation in growth rate between higher plants: a search for physiological causes and ecology consequences. Advances in Ecology Research, San Diego, v. 23, n. 1, p. 187-261, 1992. 
LIMA, J. F.; PEIXOTO, C. P.; LEDO, C. A. S. Índices fisiológicos e crescimento inicial de mamoeiro (Carica papaya L.) em casa de vegetação. Ciência e Agronomia, Lavras, v. 31, n. 5, p. 1358-1363, 2007.

MACHADO-FILHO, J. A. et al. Efeito da umidade do ar nas trocas gasosas em folhas de mamoeiro (Carica papaya L.) cultivado em condições de campo. Bragantia, Campinas, v. 65, n. 2, p. 185-196, 2006.

MAGUIRE, J. D. Speeds of germination-aid selection and evaluation for seedling emergence and vigor. Crop Science, Madison, v. 2, n. 1, p. 176-177, 1962.

MARCOS FILHO, J. Fisiologia de sementes de plantas cultivadas. Piracicaba: Fealq, 2005.

MARTELlETO, L. A. P. et al. Cultivo orgânico do mamoeiro 'Baixinho de Santa Amália' em diferentes ambientes de proteção. Revista Brasileira de Fruticultura, Jaboticabal, v. 30, n. 3, p. 662-666, 2008.
MARTELLETO, L. A. P. et al. Expressão da esterilidade feminina e capeloidia em mamoeiro sob diferentes ambientes de cultivo protegido. Revista Brasileira de Fruticultura, Jaboticabal, v. 33, n. 4, p. 1185-1193, 2011.

REIS, F. O.; CAMPOSTRINI, E. Trocas gasosas e eficiência fotoquímica potencial em mamoeiro do grupo 'Formosa' cultivado em condição de campo. Bragantia, Campinas, v. 67, n. 4, p. 815-822, 2008.

SERRANO, L. A. L.; CATTANEO, L. F. O cultivo do mamoeiro no Brasil. Revista Brasileira de Fruticultura, Jaboticabal, v. 32, n. 3, p. 657-959, 2010.

SOCOLOWSKI, F.; VIEIRA, D. C. M.; TAKAKI, M. Massa das sementes de Tecoma stans L. Juss. ex Kunth (Bignoniaceae): efeitos na emergência e desenvolvimento de suas plântulas no sol e na sombra. Biota Neotropica, Campinas, v. 11, n. 2, p. 171-178, 2011. 